\title{
The $X$-ray spectrum of the supernova remnant 1E 0102.2-7219
}

\author{
A. P. Rasmussen ${ }^{1}$, E. Behar ${ }^{1}$, S. M. Kahn ${ }^{1}$, J. W. den Herder ${ }^{2}$, and K. van der Heyden ${ }^{2}$ \\ 1 Columbia Astrophysics Laboratory, 550 West 120th Street, New York, NY 10027, USA \\ 2 University of Utrecht \& Space Research Organization of The Netherlands, Sorbonnelaan 2, 3584 CA Utrecht, \\ The Netherlands
}

Received 4 October 2000 / Accepted 10 November 2000

\begin{abstract}
In this letter we present the soft X-ray $\left(\begin{array}{lll}5-35 & \AA\end{array}\right)$ spectrum of the supernova remnant (SNR) 1E 0102.2-7219 in the Small Magellanic Cloud, acquired by the reflection grating spectrometers (RGS) aboard ESA's XMM-Newton Observatory. Because the RGS features a large dispersion angle, spatial-spectral confusion is suppressed even for moderately extended $\left(\Delta \theta \sim 2^{\prime}\right)$ sources. Consequently, these data, along with the spectrum of N132d (Behar et al. 2001), provide what are probably the most detailed soft X-ray spectrum of entire SNRs. The diagnostic power of performing spectroscopy using groups of emission lines from single ions is demonstrated. In particular, the bright Lyman and helium series lines for light elements (C VI, O viI, O viII, Ne IX \& Nex) show peculiar ratios, where the values $[1 s-n p] /[1 s-(n+1) p]$ are systematically weaker than expected for electron impact excitation close to ionization equilibrium, indicating nonequilibrium ionizing (NEI) conditions in the source. The well known temperature diagnostics $G\left(T_{\mathrm{e}}\right)=(i+f) / r$ of helium-like triplets (O VII \& Ne IX) confirm this suggestion, with values that are inconsistent with ionization equilibrium. The temperatures implied are well above the maximum emission temperature $T_{\mathrm{m}}$ for each ion, and consistent with a purely ionizing plasma. The density diagnostics $R\left(n_{\mathrm{e}}\right)=f / i$ meanwhile, are consistent with the low density limit, as expected.
\end{abstract}

Key words. atomic processes - line: formation - ISM: individual objects: 1E 0102.2-7219 - galaxies: magellanic clouds - X-rays: ISM

\section{Introduction}

The supernova remnant $1 \mathrm{E} 0102.2-7219$ (E0102) is a bright, oxygen-rich remnant located in the Small Magellanic Cloud (SMC), approximately $60 \mathrm{kpc}$ distant. Although it is young ( 1000 years old) and clearly very energetic, it has no bright emission lines of $\mathrm{Fe}$ nor significant continuum above $5 \mathrm{keV}$. It has received substantial attention recently, with the availability of high quality Chandra data (Gaetz et al. 2000; Hughes et al. 2000) as well as Hubble Space Telescope data (Blair et al. 2000). Its ionization structure in terms of spatial stratification was clearly seen for the first time with Chandra (Gaetz et al. 2000), and details of the outgoing blast wave's kinematics and emission spectrum were used to argue that a large fraction of E0102's energy is spent toward cosmic ray acceleration (Hughes et al. 2000). It was also observed with the High Energy Transmission Grating Spectrometer (HETGS) aboard Chandra to explore the spatially resolved line of sight velocity structures to isolated emission lines in the spectrum, which help to elucidate the initial geometry and mass distribution of the initial

Send offprint requests to: A. P. Rasmussen,

e-mail: arasmus@astro.columbia.edu supernova event, as well as probe the kinetics of various spectrally resolved components making up the SNR structure (Flanagan 2000).

In our work, we concentrate on the high resolution $\mathrm{X}$-ray spectroscopy afforded by the RGS (den Herder et al. 2001) aboard XMM-Newton (Jansen et al. 2001), which has nearly nominal spectral resolution, even for moderately extended objects such as E0102.

\section{Observation and data analysis}

E0102 was observed early in XMM-Newton's Calibration phase, during revolution 0065 (16 April, 2000) for a total of $42.8 \mathrm{ksec}$. All three EPIC (Turner et al. 2001, Struder et al. 2001) instruments and both RGS (den Herder et al. 2001) instruments were operated simultaneously. Here, our primary focus is on the high resolution X-ray spectrum of E0102, and we restrict attention to the RGS data ${ }^{1}$.

The data were processed using custom software, originally developed for the analysis of RGS ground calibration data, which is nearly identical in function to the RGS branch of the Science Analysis System (SAS). Telemetered

\footnotetext{
1 A separate letter on the EPIC observation of E0102 is included in this volume (Sasaki et al. 2000).
} 
CCD events were read in, frame by frame for each CCD node, and were offset corrected on a pixel by pixel basis using median readout maps, compiled from about 40 DIAGNOSTIC (den Herder et al. 2001) images per CCD chip. This process nearly eliminates flickering pixels from the dataset. Gain and CTI corrections were performed to align the signal/energy scale across all CCD readouts. Then, event reconstruction was performed on connected pixels containing significant signal, and the composite event signals were calculated by summing up signals from individual pixels.

The standard event grade combinations used were those which fall within a $2 \times 2$ pixel region, where two pixels diagonally opposed to one another within the $2 \times 2$ were not grouped into composites. The event coordinates were then mapped into focal plane angular coordinates (dispersion and cross-dispersion) ${ }^{2}$. Using the star tracker attitude history updates for the revolution, the coordinates of the source, and a preliminary boresight axis, aspect corrections were applied to the focalplane event angular coordinates ${ }^{3}$.

Events were extracted in the dispersion-pulseheight plane with masks utilized by the response matrix generator. Surviving events were then windowed in the focal plane, where background subtraction was performed using background sampling regions off to one side of the illumination pattern in the cross-dispersion direction ${ }^{4}$. The size of the spatial extraction was $58^{\prime \prime}$ in the cross-dispersion direction, which comfortably includes the entire spectrum. A spectrum file was created by computing the background corrected countrates in each dispersion channel. Finally, a response generator was used to produce an observation specific response matrix that provided an array of nominal wavelength/energy values corresponding to each channel in the spectrum file. Line fluxes were estimated by comparing an emission model, folded through the response matrix, directly to the spectrum in the usual manner. This procedure was performed for first $(m=-1)$ and second $(m=-2)$ orders for each spectrometer.

To reduce the contamination from sporadic background rate fluctuations in which the RGS counting rate doubles from the quiescent rate of about $1 \mathrm{cts}^{-1} \mathrm{RGS}^{-1}$, a set of good time intervals (GTIs) were chosen to filter out the high background data. This reduced the effective exposure time from 37.9 to $29.7 \mathrm{ksec}$ for both RGS instruments.

\footnotetext{
${ }^{2}$ The dispersion coordinates are based on the known geometry of the RGS within XMM-Newton (den Herder et al. 2001).

3 Based on attitude history updates of the star tracker system, the spacecraft pointing was extremely steady, drifting only $0.1^{\prime \prime}$ and $0.2^{\prime \prime}$ (rms) in the dispersion and cross-dispersion axes, respectively, over the observation. The respective peakto-peak attitude swings were only $0.5^{\prime \prime}$ and $0.7^{\prime \prime}$.

4 The target was $\sim 1^{\prime}$ off-axis in cross-dispersion, so the spectrum illuminated the RGS focalplane camera on one side of the CCD array. Consequently, background sampling was performed in an asymmetric manner.
}

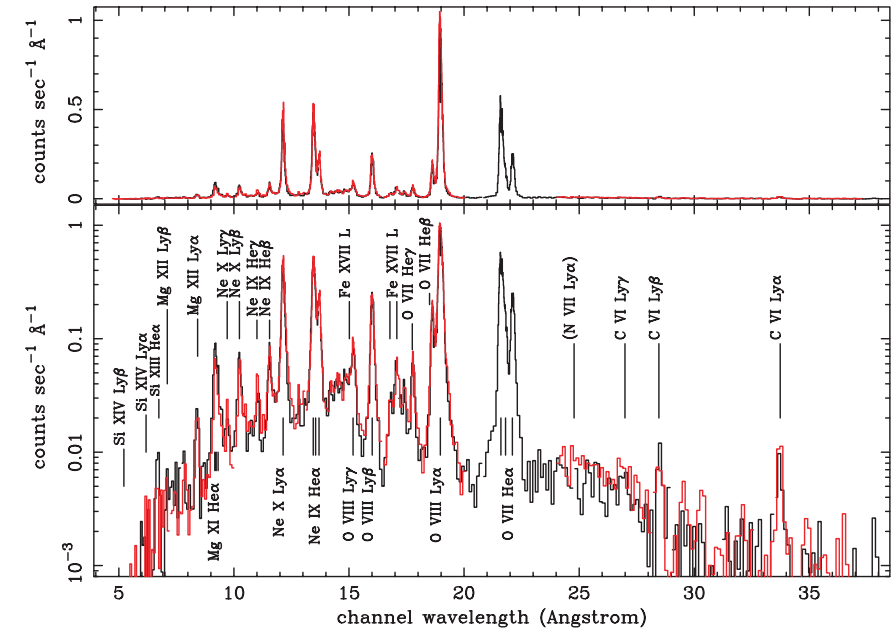

Fig. 1. The first order $(m=-1)$ RGS spectrum of E0102. The data obtained from the two RGS instruments are plotted separately (RGS1 in black, RGS2 in red). The 20-24 $\AA$ gap in the RGS2 spectrum is due to failed electronics for CCD 4 in that instrument. For clarity, the data are provided in both linear and logarithmic scales. Identifications of the principle lines are provided

The windowed background contribution to the $(m=-1)$ total countrate for this extraction was nearly flat, at $2.310^{-5} \mathrm{cts}^{-1} \mathrm{chan}^{-1}$ or, equivalently, $2.310^{-3} \mathrm{cts}^{-1} \AA^{-1}$ at $15 \AA^{5}$.

\section{The soft $X$-ray spectrum of $1 E$ 0102.2-7219}

Using the analysis procedure described above, we generated the RGS spectra shown in Figs. 1 and 2. The spectrum is dominated by a small number of emission lines, the brightest of which are prominent transitions of highly stripped light ions of $\mathrm{C}, \mathrm{O}, \mathrm{Ne}$ and $\mathrm{Mg}$. Nitrogen, which is cosmically less abundant than oxygen by a factor of a few, is absent in spectrum (N vII Ly $\alpha$ is expected at $24.7 \AA$ ). Only very weak Fe L lines are detected, which are dwarfed by the strong transitions of the light, even $Z$ elements. We detect bright lines from the Lyman series of $\mathrm{C}$ VI, O VIII, Ne X and Mg XII, and from the helium series of O VII, Ne IX, Mg XI and Si XIII. Most of these ions are represented by several emission lines in the spectrum, clearly resolved and measurable in this sparse spectrum. Single ion spectroscopy permits independent measurements of astrophysical quantities that affect line production, such as electron temperature and density, or opacity within the object and in other intervening material.

To synthesize the appropriate RGS response for E0102, we need to quantify the angular distribution of the $\mathrm{X}$-ray emission, which is convolved with the other quantities contributing to the line spread function. As a preliminary approximation, we used a public Chandra image (ObsIds 1231 \& 1423) as an energy independent

\footnotetext{
5 Also equivalent to $1.410^{-4} \mathrm{cts}^{-1}$ per resolution element. This is higher than the typical background level because of the enlarged extraction volume for this target.
} 


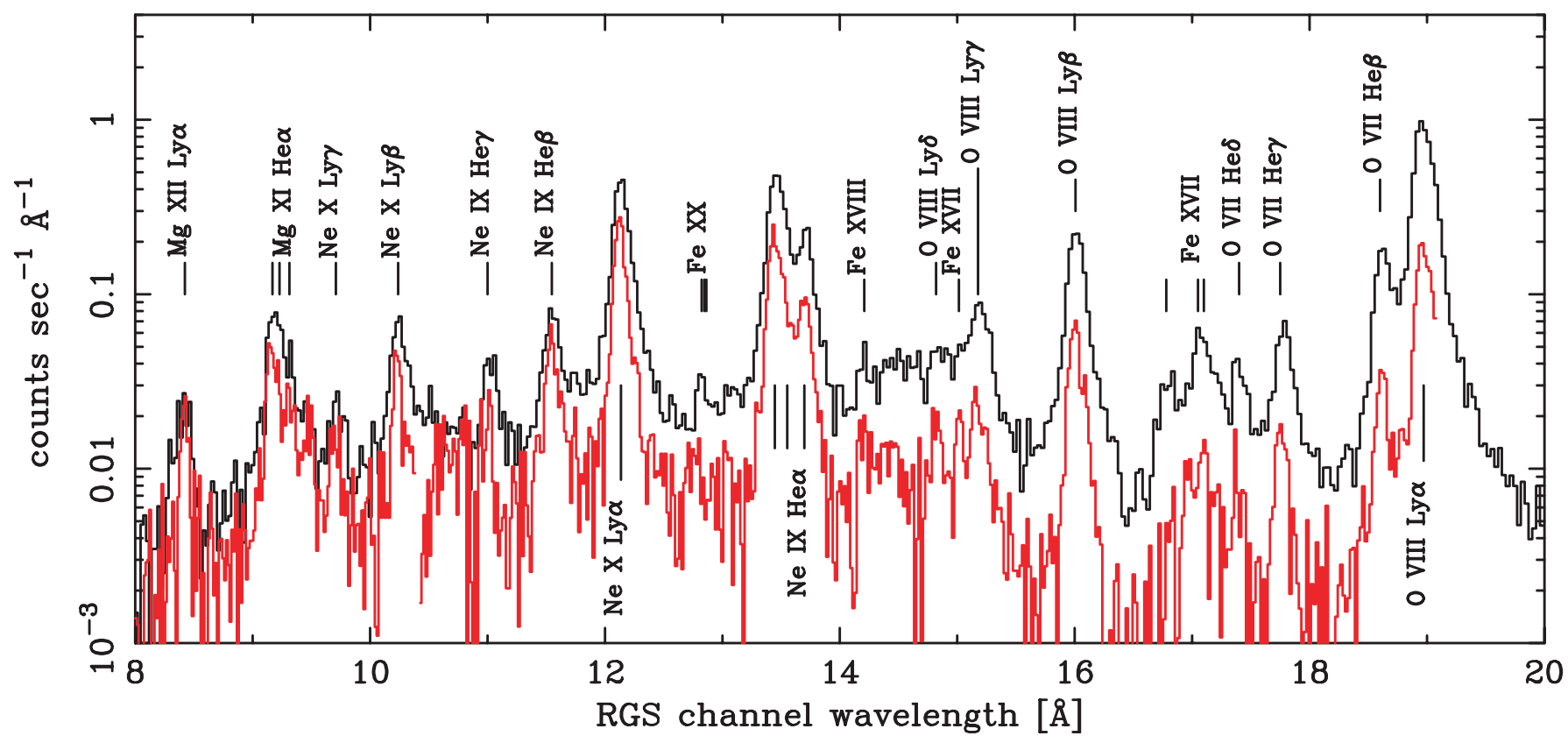

Fig. 2. Detail of the $8-20 \AA$ region of the RGS data. First and second order extractions are plotted separately $(m=-1$ in black, $m=-2$ in red) to facilitate line identification. The data from the two spectrometers have been averaged for each order extraction. The higher spectral resolution and resilience to source extent in is clearly seen in $m=-2$, where some line complexes blended in $m=-1$ are resolved

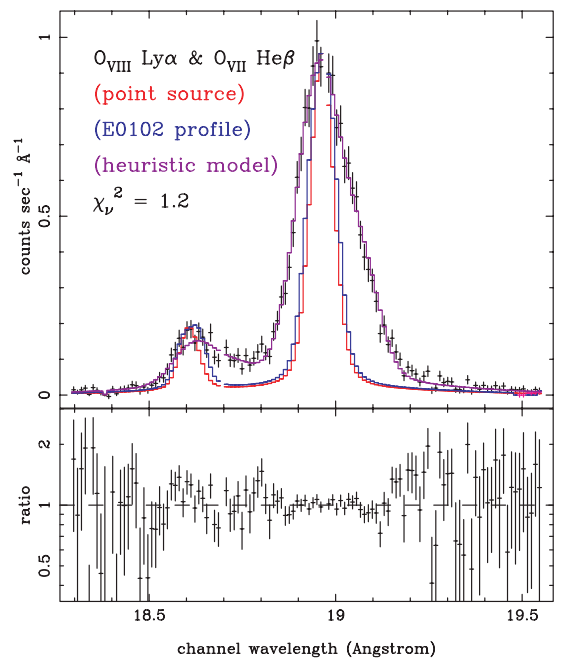

Fig. 3. Illustration of the line profile used for estimating line fluxes. The plot compares the point source line spread function for RGS1, the approximate monochromatic line profile based on E0102's angular distribution (derived from the Chandra image), and an heuristic wavelength broadening template function that is applied in addition to the angular distribution

distribution, characterized by $\sim 20^{\prime \prime}$ half power width (HPW) and $\sim 33^{\prime \prime}$ FWHM. The image was collapsed along the dispersion axis according to the roll of $X M M$ Newton during the observation. This one dimensional distribution was used as input to the response generator. Comparing the prediction to the brightest, isolated emission line ( $\mathrm{O}$ vIII $\mathrm{Ly} \alpha)$, we see that the prediction is far narrower than the data, indicating a velocity distribution resolved by the RGS (see Fig. 3). Further accurate modeling requires coupling a line of sight velocity distribution to the angular distribution, but for the time being we are able to place a lower limit to the ejecta's expansion, $v_{\exp } \geq 1350 \mathrm{~km} \mathrm{~s}^{-1}$, using the square velocity profile model of an expanding shell. This lower limit is consistent with the broadening seen in all of the lines. Any contribution from a high velocity $\left(v_{\mathrm{s}} \sim 6500 \mathrm{~km} \mathrm{~s}^{-1}\right)$ blast wave is difficult to see in this profile because it accounts for only $\sim 10 \%$ of the light (Hughes et al. 2000) and is coincident with the broad scatter distribution off of the gratings.

Spectral modeling was performed by applying this velocity distribution to all lines. While there is already evidence from Chandra that different velocity distributions are present for different charge states (Flanagan 2000), we use this distribution for the purpose of estimating line fluxes. Estimates of the line fluxes are provided in Table 1, according to the current response matrices. As indicated below, there is a known instrumental oxygen feature not included in these response matrices that we treat separately. The instrumental response and effective area are described briefly in a separate article (den Herder et al. 2001).

We attempt to correct, at least partially, for effects of intervening absorption and the known oxygen edge referred to above. Figure 4 shows the correction function utilized to estimate line fluxes from the source corrected for these effects. We adopted a neutral Galactic column of $N_{\mathrm{H}}=810^{20} \mathrm{~cm}^{-2}$ from other estimates toward the SMC (e.g., Blair et al. 2000), and also an instrumental oxygen photoelectric absorption profile characterized by $\tau_{\max }=0.2$. The curve was used to correct the nominal line fluxes listed in Table 1 and emission line ratios for 
Table 1. Estimates of line fluxes at the telescope aperture. Wherever possible, line fluxes were determined using the two spectrometers separately and the $90 \%$ confidence limits are also listed. For each emission line or complex, the RGS1 fluxes are given in the first row, followed by the RGS2 derived fluxes. Systematic differences between the spectrometers may be seen by the discrepancies in derived flux values. Because the calibration of the RGS instruments is ongoing, these flux values are uncertain by $\sim \pm 10 \%$. Also, the absolute flux in the O vII $\mathrm{K} \alpha$ is systematically underestimated by about $20 \%$, due to the presence of an instrumental oxygen absorption edge nearby. The largest discrepancy seen $(\sim 30 \%)$ is in the flux values for the Mg XI, is about twice as large as expected. The top part of the table gives the total line intensities in the $\mathrm{K} \alpha, \mathrm{K} \beta$ and $\mathrm{K} \gamma$ transitions for each ion; the bottom part gives the breakdown between the resonance $(r)$, intercombination $(i)$ and forbidden $(f)$ transitions for the helium like species O VII, Ne IX and $\mathrm{Mg}$ XI. All fluxes are in units of $10^{-4}$ phot $\mathrm{cm}^{-2} \mathrm{~s}^{-1}$

\begin{tabular}{|c|c|c|c|}
\hline \multirow[t]{2}{*}{ IIon } & \multicolumn{3}{|c|}{$\overline{\text { Transition }}$} \\
\hline & $\mathrm{K} \alpha^{c}$ & $\mathrm{~K} \beta^{c}$ & $\mathrm{~K} \gamma^{c}$ \\
\hline \multirow[t]{2}{*}{ C vi } & $0.94_{-.16}^{+.38}$ & $0.69_{-.11}^{+.17}$ & $\mathrm{NA}$ \\
\hline & $1.01_{-.20}^{+.20}$ & $0.52_{-.13}^{+.13}$ & $\mathrm{NA}$ \\
\hline \multirow[t]{2}{*}{$\mathrm{OVVII}^{a}$} & $30.0_{-.68}^{+.66}$ & $4.12_{-.30}^{+.30}$ & $2.02_{-.19}^{+.19}$ \\
\hline & $\mathrm{NA}$ & $4.88_{-.30}^{+.30}$ & $2.17_{-.17}^{+.18}$ \\
\hline \multirow[t]{2}{*}{ O viII } & $29.8_{-.68}^{+.68}$ & $6.70_{-.32}^{+.31}$ & $3.07_{-.22}^{+.21}$ \\
\hline & $32.8_{-.65}^{+.65}$ & $7.39_{-.30}^{+.30}$ & $3.49_{-.21}^{+.21}$ \\
\hline \multirow[t]{2}{*}{ NeIX } & $20.4_{-.70}^{+.63}$ & $2.44_{-.21}^{+.21}$ & $1.16_{-.15}^{+.16}$ \\
\hline & $21.2_{-.53}^{+.54}$ & $2.63_{-.22}^{+.22}$ & $1.68_{-.17}^{+.17}$ \\
\hline \multirow[t]{2}{*}{$\mathrm{Nex}$} & $12.5_{-.43}^{+.43}$ & $3.04_{-.27}^{+.27}$ & $0.87_{-.13}^{+.23}$ \\
\hline & $14.3_{-.45}^{+.46}$ & $2.44_{-.21}^{+.21}$ & $1.08_{-.23}^{+.09}$ \\
\hline \multirow[t]{2}{*}{$\mathrm{Mg} \mathrm{XI}^{b}$} & $5.28_{-.37}^{+.31}$ & $\mathrm{NA}$ & $\mathrm{NA}$ \\
\hline & $3.52_{-.26}^{+.26}$ & NA & NA \\
\hline \multirow[t]{3}{*}{ Mg XII } & $1.29_{-.21}^{+.26}$ & NA & NA \\
\hline & $1.22_{-.17}^{+.17}$ & NA & NA \\
\hline & He K $\alpha(r)$ & $\mathrm{He} \mathrm{K} \alpha(i)$ & He $\mathrm{K} \alpha(f)$ \\
\hline \multirow[t]{2}{*}{$\mathrm{OVII}^{a}$} & $19.5_{-.69}^{+.66}$ & $2.48_{-.39}^{+.41}$ & $8.00_{-.42}^{+.40}$ \\
\hline & $\mathrm{NA}$ & NA & $\mathrm{NA}$ \\
\hline \multirow[t]{2}{*}{ NeIX } & $13.8_{-.70}^{+.63}$ & $1.12_{-.54}^{+.59}$ & $5.48_{-.59}^{+.44}$ \\
\hline & $13.6_{-.53}^{+.54}$ & $1.54_{-.49}^{+.49}$ & $6.08_{-.35}^{+.36}$ \\
\hline \multirow[t]{2}{*}{$\mathrm{Mg} \mathrm{XI}^{b}$} & $3.84_{-.37}^{+.31}$ & $0_{-0}^{+.48}$ & $1.44_{-.26}^{+.29}$ \\
\hline & $2.78_{-.26}^{+.26}$ & $0_{-0}^{+.38}$ & $0.74_{-.18}^{+.19}$ \\
\hline
\end{tabular}

${ }^{a} \mathrm{O}$ viI $\mathrm{K} \alpha$ fluxes are underestimated here by about $20 \%$.

${ }^{b}$ Discrepancy in Mg XI K $\alpha$ fluxes is currently not understood. ${ }^{c} \mathrm{~K} \alpha, \mathrm{K} \beta$ and $\mathrm{K} \gamma$ represent the $1-2,1-3$ and $1-4$ transitions, respectively.

individual ions were estimated. These are displayed in the lower two plots of Fig. 4.

The estimated emitted line ratios have not been corrected for any additional intervening absorption, e.g., contributions from the SMC. Any such column density would systematically affect the line ratios at long wavelength, which may be reflected by the low value for the $\mathrm{C}$ VI $\alpha / \beta$ ratio. As shown in the figure, the predicted intraseries ratios $\alpha / \beta$ and $\beta / \gamma$ for heliumlike and hydrogenlike ions are dependent on $T_{\mathrm{e}}$, but approach their minimum
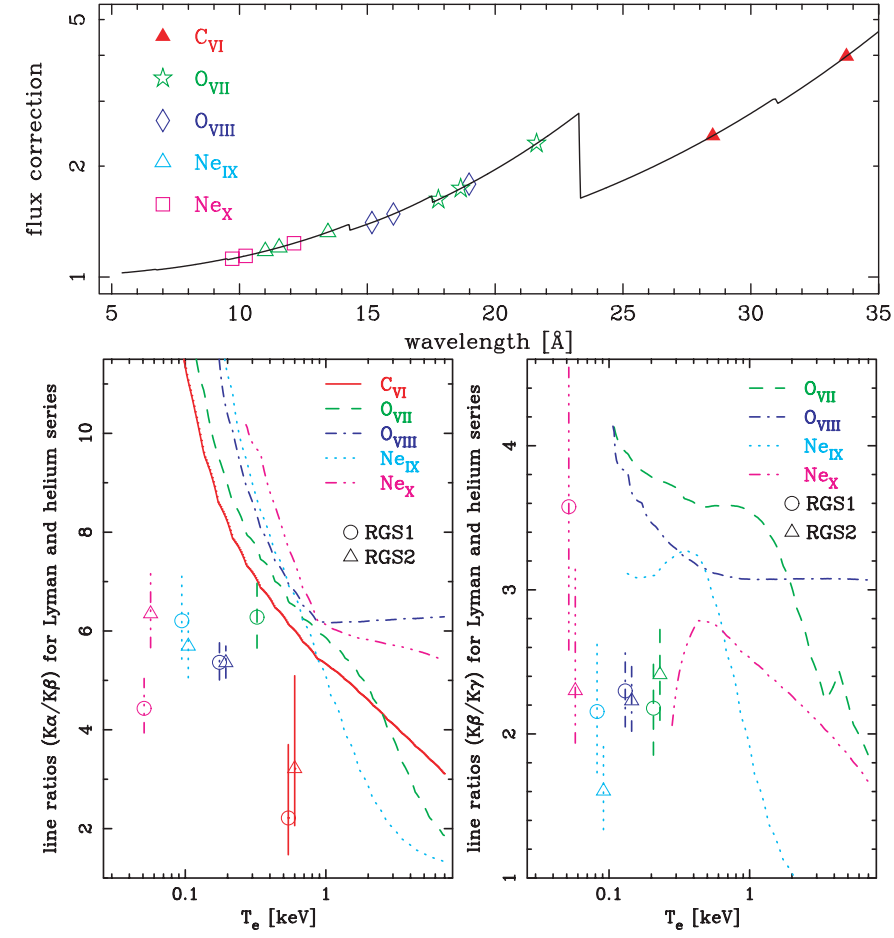

Fig. 4. Estimated resonance line ratios emitted at the source. Line emission ratios for individual ions are calculated by correcting the values in Table 1 according to the Galactic absorption and the additional instrumental oxygen absorption edge not implemented in the current response matrices. The correction function used is shown in the upper plot. Estimated flux ratios are given in the lower two plots, with the confidence limits associated with them for each RGS. The expected dependences of these ratios on electron temperature $T_{\mathrm{e}}$ are provided in the curves. We used the APEC (Smith et al. 1999) CIE model to produce these, so we expect slightly different temperature dependences for NEI cases. The lower left plot shows the ratios of $\operatorname{Ly} \alpha / \mathrm{Ly} \beta$ ( $\mathrm{C}$ VI, O VIII \& $\mathrm{Nex}$ ) and $\mathrm{He} \alpha(r) / \mathrm{He} \beta$ (O viI \& Ne IX); the lower right plot gives Ly $\beta / \mathrm{Ly} \gamma(\mathrm{O}$ VIII \& $\mathrm{Nex}$ ) and $\mathrm{He} \beta / \mathrm{He} \gamma(\mathrm{O}$ VII \& Ne IX)

values in the limit of high temperature. Our ratio estimates, compared to the current APEC (Smith et al. 1999) collisional ionization equilibrium (CIE) emission model, suggest electron temperatures $T_{\mathrm{e}}$ that are significantly higher than the maximum line emission temperatures $T_{\mathrm{m}}$ in CIE. Consistent temperature estimates are obtained for Ne IX line ratios, which yield $0.6 \leq T_{\mathrm{e}} \leq 1.0 \mathrm{keV}$. In other cases a self consistent $T_{\mathrm{e}}$ cannot be obtained in the APEC CIE model, given the estimated emission ratios. For example, O viI, derived $T_{\mathrm{e}}(\alpha / \beta)$ and $T_{\mathrm{e}}(\beta / \gamma)$ values are inconsistent by a factor of 2.5 or so. For O VIII, the APEC curves do not intersect the line ratio confidence limits.

We performed sample line ratio calculations using HULLAC (Bar-Shalom et al. 1998) in which the plasma is underionized as compared to CIE, in order to simulate the purely ionizing case. These ratios were within $\sim 10 \%$ of the APEC ratios for the O VIII case for both $\alpha / \beta$ and $\beta / \gamma$. For O VII however, the HULLAC line ratios deviate from the APEC CIE values and assymptotically 


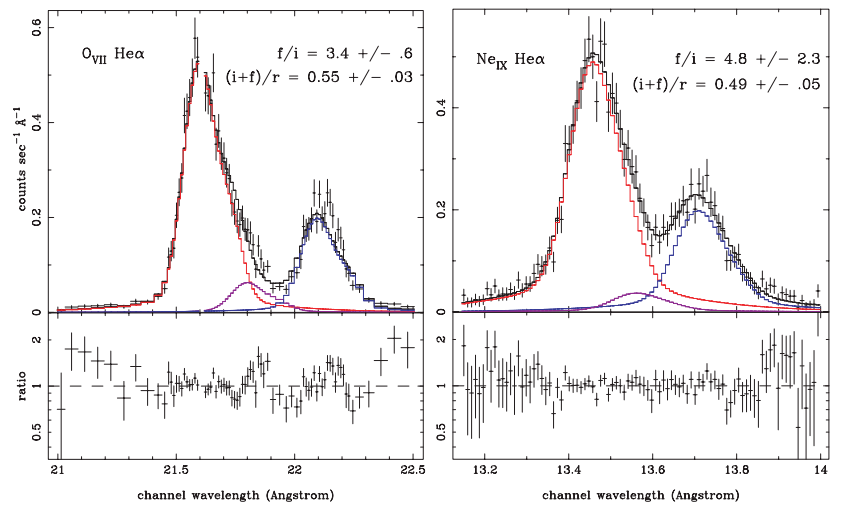

Fig. 5. Derivation of the $R$ (density) and $G$ (temperature) line ratio diagnostics for O VII (left) and NeIX (right). The data were fit using three line features, where we have used the heuristic velocity distribution function derived from $\mathrm{O}$ vIII Ly $\alpha$ as a template to describe each (see Fig. 3). One parameter adjusted the ensemble line position (relative line positions were fixed), and the three line intensities were allowed to vary to fit the data. Individual contributions from the $f, i$ and $r$ lines are shown. The $\chi_{\nu}^{2}$ values are 1.4 and 0.9 for the O VII and Ne IX triplets, respectively

approach 4.8 and 2.5 for the $\alpha / \beta$ and $\beta / \gamma$ ratios, respectively. Comparing the O viI line ratios to the HULLAC values, we obtain a self consistent temperature range of $0.35 \leq T_{\mathrm{e}} \leq 0.7 \mathrm{keV}$. Interestingly, the line ratios for O VIII remain low as compared to either the CIE (APEC) or the ionizing (HULLAC) cases.

In spite of the angular extent of the source and the velocity broadening of the lines, we resolve the He $\alpha$ line complexes of O VII and Ne IX sufficiently to estimate contributions from the forbidden, intercombination and resonance transitions that provide robust diagnostics for the temperature and density of the emitting medium (Gabriel \& Jordan 1969; Pradhan 1982). Some contamination from satellite line blending is present in the flux estimates of the intercombination $(i)$ and forbidden $(f)$ lines, but only at the 3 and $6 \%$ level for O VII and Ne IX, respectively (Pradhan \& Shull 1981). These effects are smaller or comparable to the line flux confidence limits and are also taken into consideration in diagnostic line ratio calculations (Pradhan 1982). Figure 5 shows the heliumlike triplets of $\mathrm{O}$ VII and Ne IX, modelled using the known line wavelengths, and the line velocity profile adopted above (Fig. 3). While the density diagnostic $R$ ratios obtained were consistent with the low density limit at the $90 \%$ confidence level, the temperature $\operatorname{diagnostic} G$ values are low. We measure at $0.55 \pm .03$ and $0.49 \pm .05$ for O VII and Ne IX respectively. It should be noted here that these small values for $G$ are inconsistent with collisional equilibrium, and require underionization, strongly suppressing photons produced in recombination channels from O VIII and Ne IX (Pradhan 1982). The electron temperatures corresponding to these ratios are approximately 0.35 and $0.65 \mathrm{keV}$ for O VII and NeIX respectively, in agreement with the $90 \%$ confidence limits derived from the resonance line ratio estimates above. In CIE conditions for these temperatures, the fractional population of these charge states whould be only $\sim 2 \%$ for both O VII and Ne IX (Arnaud \& Rothenflug 1985). The fact that consistent values for $T_{\mathrm{e}}$ are obtained for both O viI and Ne IX using all three available diagnostics, suggest that $\mathrm{K} \alpha$ resonance line suppression effects (such as absorption or resonant scattering) are small.

\section{Discussion}

The Fe L lines that we do see in the SNR most likely trace the swept up ISM rather than the ejecta of the precursor. The Fe:O abundance ratio that we measure is about 1:900, compared to $1: 15$ or so in the SMC (Russell \& Dopita 1992). With the exception of Fe, the composition of the SNR is dominated by products of He burning, the SN ejecta. The absence of emission from $\mathrm{N}$ is suggestive that any nitrogen in the progenitor had been either blown off or burned into other products.

Analysis of the heliumlike ion spectra for O VII and NeIX yields self consistent pictures for these plasmas: There exist temperatures which simultaneously yield the line ratios seen $(G, \alpha / \beta \& \beta / \gamma)$ provided that the neighboring charge states (O VIII \& Nex) are underpopulated there. The presence of bright emission lines from the hydrogenlike ions must then indicate a different volume containing them. This is in fact consistent with the Chandra findings (Gaetz et al. 2000; Flanagan 2000) that emission from O VII and O VIII appear to originate from different regions within the SNR. In the Chandra HETGS "spectroheliograms", isolated line images show that the bright O VII emission occurs significantly inside of the region emitting in $\mathrm{O}$ VIII. The inner, $\mathrm{O}$ VII region is presumably ejecta that has more recently encountered the inward moving shock than the outer O VIII region.

The emission line pattern of the OVIII Lyman series clearly requires better understanding; these ratios are better constrained and are consistent across RGS instruments, yet they are inconsistent with current electron impact excitation (EIE) models. Opacity effects to the Ly $\alpha$ flux should be negligible because of the consistency found in the O VII and Ne IX He series data.

We consider charge exchange (CE) as a possible mechanism that might contribute to produce the observed flux ratios. The emission spectral signature associated with a CE reaction generally resembles that of recombination (Wise \& Sarazin 1989), because in both mechanisms, an electron is injected into a high quantum level and then cascades. The flux ratios due to pure recombination of O IX to O viII are about 4.7 and 2.6 for $\alpha / \beta$ and $\beta / \gamma$, respectively (using HULLAC). The same ratios for EIE in the high temperature limit, are 6.2 and 3.0. Weighting the contributions from CE and EIE appropriately, it may be possible to produce the observed values of $5.4 \pm .4$ and $2.3 \pm .3$, respectively. We cannot preclude the presence of $\mathrm{CE}$ reactions involving $\mathrm{O}$ VIII in the emission line ratios of OVII, although the emission there appears generally consistent with extreme NEI conditions. 
In $\mathrm{CE}$ reactions, the exchanged electrons are injected into specific high $(n, l)$ levels according to energy conservation. Spectra from such reactions have been observed extensively in tokamak and ion beam experiments, for example, in highly ionized $\mathrm{Ar}^{+18}$ (Rice et al. 1986) and $\mathrm{O}^{+8}$ (Bliman et al. 1985). In the latter work, CE reactions of $\mathrm{O}^{+8}$ with molecular $\mathrm{H}_{2}$ yielded values of $\mathrm{Ly} \alpha / \mathrm{Ly} \beta \sim 4.2$ and $\operatorname{Ly} \beta / \operatorname{Ly} \gamma \sim 3.1$ (40\% relative error in each) and a preferential injection into $n=5$. Calculations performed for interactions with atomic hydrogen yield values with comparable variances, but all well below ratios predicted by EIE.

In order for $\mathrm{CE}$ to contribute significantly to the $\mathrm{X}$-ray line emission from a SNR, low ionization species must come into close proximity with more heavily ionized metals (such as OIX). Given that the $\mathrm{CE}$ and EIE rate coefficients are of order $\gamma_{\mathrm{CE}} \sim 10^{-6}$ and $\gamma_{\text {EIE }} \sim 10^{-11} \mathrm{~cm}^{3} \mathrm{~s}^{-1}$, respectively, CE can be important if the neutral fraction for hydrogen is greater than $\sim 10^{-5}$ or so (Wise \& Sarazin 1989). This is well above the neutral fraction expected for the hot ionized plasma where the $\mathrm{X}$-ray emission is thought to originate. In the case of E0102, the amounts of $\mathrm{H}$ and He present in the ejecta are probably very low, so the metal ion to electron ratio should be extremely high as compared to solar abundance material. Consequently, $\mathrm{CE}$ is more likely to be important in such a plasma, particularly if inhomogeneities are present and mixing occurs between regions.

There is abundant evidence that the $\mathrm{X}$-ray emitting media in SNRs are far from homogeneous. For example, [O III] emission is seen in many SNRs, including E0102 (Dopita et al. 1981) and some of the bright knots seen there correspond well to X-ray bright features (Gaetz et al. 2000). Efforts to numerically model shock-cloud interactions produce strong fragmentation, turbulent mixing action and complex filamentary structures (e.g., Xu \& Stone 1995). These general predictions should apply also to interactions between ejecta clumps and the reverse shock. The material in E0102 may be comprised primarily of metals, and cooling rates there can be enormous, so radiative shock conditions may exist even at high $T_{\mathrm{e}}$. The survivability of ejecta clumps and filaments embedded in strong shocks would consequently improve, so interfaces between hot, tenuous media and warm, denser filaments could be prevalent.

At these interfaces, one expects a steady flux of high charge state ions from the hot medium. These ions are likely to charge exchange with the lower ionization states in the clouds, giving rise to $\mathrm{X}$-ray emission lines. Since the charge exchange cross-section for the ions is considerably larger than the photoelectric absorption cross-section for the emitted line photons, the emitted line radiation should emerge and contribute to the observed $\mathrm{X}$-ray spectrum of the remnant. This process should be important if the mean intercloud separation is smaller than the characteristic distance $d$ an ion will move through the hot gas before it is collisionally excited:

$$
d=1.0 \mathrm{pc}\left(\gamma_{\mathrm{EIE}} / 10^{-11}\right)^{-1} T_{\mathrm{keV}}^{1 / 2} A_{\mathrm{AMU}}^{-1 / 2} n_{\mathrm{e}}^{-1} .
$$

The Chandra image of E0102 (Gaetz et al. 2000) exhibits significant structure on spatial scales comparable to or less than this value. It therefore seems plausible that charge exchange at cloud interfaces plays a significant role in producing the anomalous line ratios that we observe in O VIII.

Acknowledgements. This work is based on observations obtained with XMM-Newton, an ESA science mission with instruments and contributions directly funded by ESA Member States and the USA (NASA). We thank our referree, T. J. Gaetz, for insightful comments and suggestions that substantially improved this manuscript. We also thank D. W. Savin for useful discussions on charge transfer processes and resulting emission.

\section{References}

Arnaud, M., \& Rothenflug, R. 1985, A\&AS, 60, 425

Bar-Shalom, A., Klapisch, M., Goldstein, W. H., \& Oreg, J. 1998, unpublished

Behar, E., Rasmussen, A. P., Griffiths, R. G., et al. 2001, A\&A, 365, L242

Blair, W. P., Morse, J. A., Raymond, J. C., et al. 2000, ApJ, 537,667

Bliman, S., Bonnet, J. J., Montenesquieu, A. B., et al. 1985, Nucl. Instrum. Meth. Phys. Res., Sect. B, 9, 371

den Herder, J. W., Brinkman, A. C., Kahn, S. M., et al. 2001, A\&A, 365, L7

Dopita, M. A., Tuohy, I. R., \& Matthewson, D. S. 1981, ApJL, 248, L105

Flanagan, K. 2000, in X-Ray Astronomy 2000, ed. R. Giacconi, L. Stella, \& S. Serio, Publications of the Astronomical Society of the Pacific Conference Series (PASP)

Gabriel, A. H. \& Jordan, C. 1969, MNRAS, 145, 241

Gaetz, T. J., Butt, Y. M., Edgar, R. J., et al. 2000, ApJ, 534, L47

Hughes, J. P., Rakowski, C. E., \& Decourchelle, A. 2000, ApJ, in press

Jansen, F., Lumb, D., Altieri, B., et al. 2001, A\&A, 365, L1

Pradhan, A. K. 1982, ApJ, 263, 477

Pradhan, A. K., \& Shull, J. M. 1981, ApJ, 249, 821

Rice, J. E., Marmar, E. S., Terry, J. L., Kallne, E., \& Kallne, J. 1986, Phys. Rev. Lett., 56, 50

Russell, S. C. \& Dopita, M. A. 1992, ApJ, 384, 508

Sasaki, M., Stadlbauer, T. F. X., Haberl, F., Filipović, M. D., \& Bennie, P. J. 2000, A\&A, 365, L237

Smith, R. K., Brickhouse, N., Raymond, J., \& Liedahl, D. 1999, unpublished, version 1.0.1 available at http: //hea-www. harvard. edu/APEC.

Strüder, L., Briel, U. G., Dennerl, K., et al. 2001, A\&A, 365, L18

Turner, M. J., Abbey, A., Arnaud, M., et al. 2001, A\&A, 365, L27

Wise, M. W., \& Sarazin, C. L. 1989, ApJ, 345, 384

Xu, J., \& Stone, J. M. 1995, ApJ, 454, 172 